\title{
Wrist and Forearm Rotation of the DLR Hand Arm System: Mechanical Design, Shape Analysis and Experimental Validation
}

\author{
Werner Friedl, Hannes Höppner, Florian Petit and Gerd Hirzinger \\ Institute of Robotics and Mechatronics, German Aerospace Center (DLR), Wessling, Germany \\ E-mails: \{Werner.Friedl, Hannes.Hoeppner, Florian.Petit \}@dlr.de
}

\begin{abstract}
The DLR Hand Arm System is based upon the variable stiffness concept which has been recently developed to improve impact robustness and energy efficiency of modern robots. This paper continues the work on the bidirectional antagonistic variable stiffness (BAVS) joint concept which is an extension of antagonistic joints. Three mechanical setups utilizing different spring and cam disc combinations to implement a desired torque-stiffness characteristic are analyzed. Two BAVS joint solutions as used for the wrist and forearm rotation of the DLR Hand Arm System are presented. Furthermore in the experimental section torque-deflection calibration and drive redundancy are validated.
\end{abstract}

\section{INTRODUCTION}

Recent research led to the development of the variable stiffness joint technology for robots. As reported in [1] DLR has developed the biologically motivated variable stiffness robot arm called the Hand Arm System (HASy) in the past. The robot provides 26 degrees of freedom (DoF), where 19 DoF are mounted in the hand and seven DoF in the fore- and upper arm integrated with all electronic devices. Several Variable Stiffness Actuators (VSA) used to adjust the position and stiffness simultaneously have been analyzed by various researchers [2] [3] [4] [5] [6] [7]. Using VSA provides several benefits as e.g. the intrinsic compliance gives the possibility to store mechanical energy in the joints similar to the human. The low pass force filter properties of elastic elements are especially relevant for robustness reasons in the hand. Furthermore, the energy storage property can be used for highly dynamic tasks as throwing a ball or during walking. Passive compliance is also discussed in the context of human robot safety [8].

While the VSA idea is similar for all the mentioned joint prototypes, the mechanical implementation varies widely and the evaluation of different VS joints is ongoing research [9]. Therefore also multiple different VS joints have been implemented in the DLR Hand Arm system. For the 19 DoF of the hand [10] an antagonistic principle is used similar to the human hand with its elastic tendons. The arm joints 1-4, namely the elbow and the three shoulder joints, are implemented by Floating Spring Joints (FSJ) [11]. A principle called the Bidirectional Antagonistic Variable Stiffness (BAVS) [12] concept has been used for both the forearm and the wrist joints. The objective of this paper is to introduce the developed BAVS joints used in the HASy.

This paper is structured in the following way. We begin by evaluating the requirements and the benefits of the BAVS design compared to other VSA principles in the context of the DLR Hand Arm System. Next we analyze the results of a desired torque-stiffness curve for the mechanism cam discs which are responsible for the VS properties of the joint. We will focus on different combinations of cam discs and linear springs for BAVS joints followed by the resulting mechanical design of the forearm and both wrist joints. Finally we will validate the capability of BAVS joints with first measurements and show results of a implemented automatic stiffness adaption.

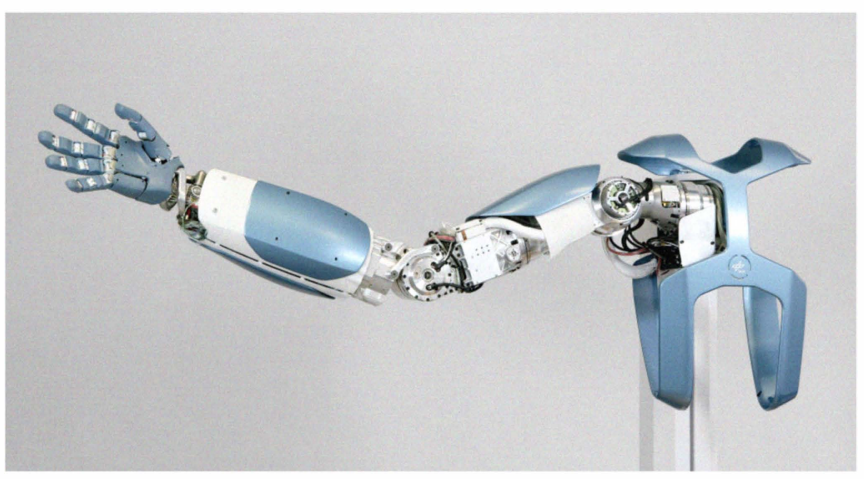

Fig. 1. The DLR Hand Arm System.

\section{BAVS JOINT}

The DLR Hand Arm system incorporates several different joint types. The BAVS joint principle was used for the implementation of the wrist and forearm joints. This choice follows from the requirements as presented in the following.

\section{A. Requirements}

Due to the location of the wrist joints and the forearm rotation joint, the requirements compared to other HASy joints are different:

- Wrist: In order to achieve the same size as the human wrist both wrist joint actuators can not be placed coincident to the joint axes, but are placed in the forearm close to the elbow. Thus the torque of the motors had to be transfered from the forearm to the wrist, similar to the power transfer implemented by tendons in the finger joints.

- Wrist: Furthermore the mechanical power transfer had to be as stiff as possible in order to achieve a direct 


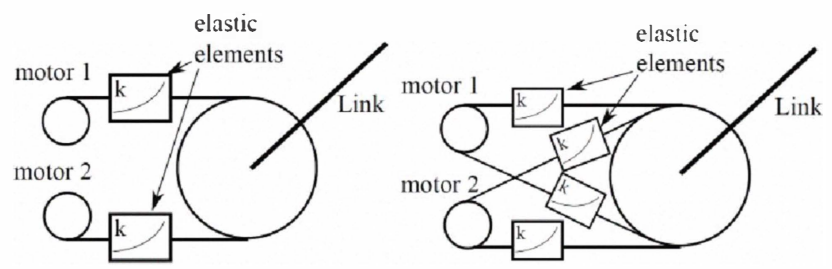

Fig. 2. Antagonistic and BAVS drive principle using tendons.

coupling of the actuators to the wrist. This is important as the motion of the fingers is coupled to the wrist to some extend. Thus rather flexible tendons can not be used.

- Forearm: A main challenge for the forearm rotation joint is to transfer the electronic power supply cable, water cooling tubes, and the communication bus cable to the forearm, while allowing a rotation range of $180^{\circ}$.

- On the other side the power to size ratio of the forearm and both wrist joints had to be optimized because of the limited space in the 42 actuators containing forearm.

- The smaller inertia and weight that has to be supported by the wrist and forearm rotations ask for lower torque capability compared to the shoulder and elbow joints.

More general requirements that also other vSA joints must fulfill are the need of low friction and low inertia of the mechanical design in order to achieve high dynamic capabilities.

The use of the BAVs joint concept was chosen to be best suited for the requirements. The following sections will go into details of this choice.

\section{B. BAVS principle}

Before going into design details, the main properties of the BAVS principle are given. In Fig. 2 the standard antagonistic principle and the BAVS principle are shown for comparison. The way of changing stiffness and position is the same for both: Co-contracting the springs by the motors leads to a change in stiffness, while a synchronous motion of the motors applies a torque at the joint. The essential difference is that both motors of the BAVS joint can push and pull on the joint. Thus the maximum torque of the joint equals the sum of the torques of both motors

$$
\tau=\tau_{1}+\tau_{2} . \quad\left|\tau_{i}\right| \leq \tau_{\text {stall }} \forall i
$$

where $\tau_{1}$ and $\tau_{2}$ are the torques provided by the two motors. The stall torque $\tau_{\text {stall }}$ is the maximum torque of one motor. This capability of the BAVS principle to use the motors in a supporting way is referred to as the helping mode. The overall link stiffness $k$ equals the sum of the stiffness $k_{1}\left(\tau_{1}\right)$ and $k_{2}\left(\tau_{2}\right)$ of each motor spring unit.

$$
k=k_{1}\left(\tau_{1}\right)+k_{2}\left(\tau_{2}\right) \quad 0 \leq k_{i}\left(\tau_{i}\right) \forall \tau_{i}
$$

Figure 4 shows the BAVS joint setup with one spring and two symmetric cam discs. The two characteristic modes are shown. In Fig. 4 b) the joint is in the helping mode, where both cam discs provide a torque in the same direction. The

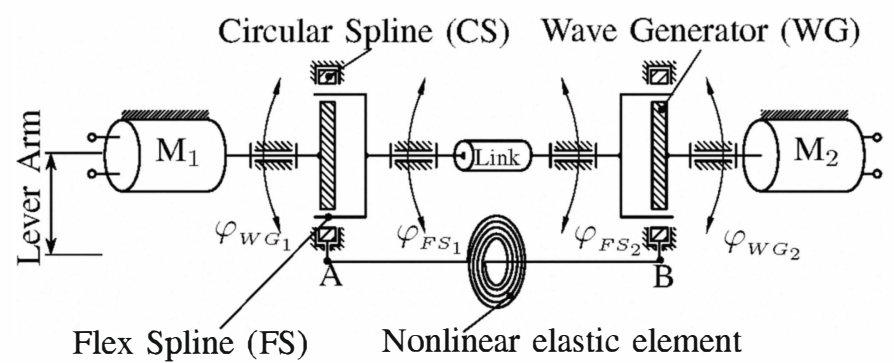

Fig. 3. The mechanical implementation of the BAVS principle on the forearm rotation. Harmonic drives are used to incorporate the nonlinear elastic elements and to transfer the power to the link. For the differnent implementation of the nonlinear elastic element see Fig. 5

normal mode with opposing torques provided by the cam discs is shown in Fig. $4 \mathrm{c}$ ).

Compared to the FSJ (where one big motor changes the position and a small motor the stiffness of the joint) and the antagonistic principle, the bidirectional antagonisms leads to a high power to size ratio as it is primarily required for the forearm and both wrist joints.

\section{Mechanism setup}

The requirement of high stiffness of the wrist in order to avoid unmeant wrist movement waives the use of a tendon based VSA mechanism. Instead, the three-part setup of harmonic drive gears is used to couple both the motors to the elastic elements and the drive side of the joint, see Fig. 3.

The joint works in the following way see Fig. 3. The wave generators are connected to the motors. A spur gear couples the flex splines with the link output. Both circular splines are connected to the nonlinear elastic element. Moving both motors in the same direction leads to a change in position of the link (the spur-wheel). Moving the motors in opposite direction results in no link motion as the flex splines are blocking each other via the link. The torque applied at the circular splines results in a tensioning of the nonlinear elastic element. By blocking the motors, any external motion of the link will load the spring element. Thus a natural torque-angle-relation similar to that of the human arm with a nonlinear increase of torque [13] can be achieved.

The nonlinearity of the stiffness elements is achieved by nonlinear cam discs which are actuating one or more linear springs. The shape of the cam discs allows to influence the torque-stiffness curve of the joint.

In [12] the effect of torque-stiffness curves on the stiffness variation during the helping mode is analyzed.

\section{BAVS SHAPE ANALYSIS}

The proposed mechanical setup presented in Section II-C provides mainly two design parameters. First, the number of linear springs (one or two) and second the shape of the cam discs (symmetric or unsymmetric). Three different setups are analyzed in the following.

The simplest setup is the single spring solution using only one linear spring, as shown in Fig. 5 a). The double spring 


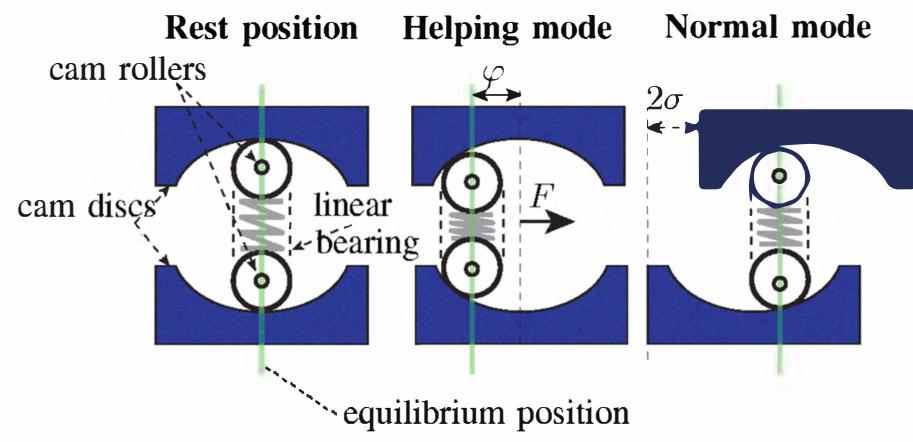

Fig. 4. A BAVS joint implementation using cam discs: In a) the mechanism is in rest position. In b) the joint is deflected with by external torque and is in the helping mode. In c) the joint is in the normal mode with a stiff preset.

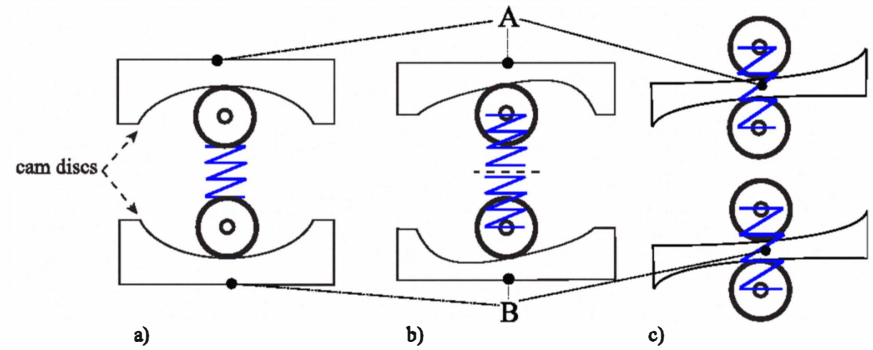

Fig. 5. Three different mechanical BAVS solutions as a combination of linear springs and cam discs. A and B are the connecting flanges for the circular spline of the gear

solution uses two instead of only one spring, but in the same setting with two cam discs, see Fig. 5 b). The third analyzed setup is shown in Fig. 5 c) and contains two springs and four cam discs.

All the following joints are designed to reach a maximum torque of $8 \mathrm{Nm}$, which is twice the stall torque of one motor $\left(\tau_{\max }=2 \cdot \tau_{\text {stall }}=8 \mathrm{Nm}\right)$, at a deflection angle of $15^{\circ} . \mathrm{A}$ linear spring with the stiffness of $22.1 \mathrm{kN} / \mathrm{m}$ has been used.

\section{A. Single spring solution}

The simplest possible solution is a combination of one spring and two symmetric cam discs (see Fig. 5) and requires the least construction space. The torque applied by both symmetric cam discs to the joint can be calculated by

$$
\begin{array}{r}
\tau=c\left(f_{c 1}(\phi+\sigma)+f_{c 2}(\phi-\sigma)\right) \\
r\left(f_{c 1}^{\prime}(\phi+\sigma)+f_{c 2}^{\prime}(\phi-\sigma)\right),
\end{array}
$$

where $\phi$ is the deflection angle of the joint, $\sigma$ is the pretension angle of the cam discs, $c$ the spring rate, $r$ the lever arm between cam disc shape and rotation center of cam disc, and $f_{c 1}, f_{c 2}$ and $f_{c 1}^{\prime}, f_{c 2}^{\prime}$ are mathematic function which describe the relations between deflection angle of the cam discs and deflection of the springs and their derivatives. The stiffness of the joint is the derivative of the torque (3) with respect to the deflection $\phi$.

1) Symmetric cam discs: For symmetric shape design the stiffness around zero deflection $\phi=0^{\circ}$ is low, as the cam rollers radius limits the cam disc shape. This is because of to guarantee proper rolling of the cam rollers, the curvature of the cam discs has to be less than the radius of the cam rollers. Thus for a symmetric cam disc shape the constraint $f_{c}(0)=f_{c}^{\prime}(0)=0$ is essential for avoiding discontinuity.

Another disadvantage results from the one spring solution. The range of the joint torque for a pretensioned joint is limited, because of the maximum spring force will only be available if both cam discs are almost fully deflected. As a result at best only about a quarter of the maximum torque is available if the joint is in normal antagonistic mode. This is illustrated by Fig. 6 top, where the torque-stiffness relation is depicted for the single spring solution with symmetrical shape design. The dashed curve shows a pretension of $\sigma=50 \%$ what results in approximately a quarter of the maximum achievable torque. Furthermore, the graph can be interpreted as follows: The step size between the curves equals an increase of pretension of $10 \% \sigma_{\max }$. Additionally the curve where $\tau_{\text {stall }}$ of one of the two motors is reached is printed bold in the figures because this curve equals the boundary between normal and helping antagonistic mode (see also [12] Chapter IV.A). The lowest curve equals the non pretension mechanism $\left(\tau_{\max }=2 \cdot \tau_{\text {stall }}\right)$. The highest curve equals a pretension of $\sigma=90 \%$.

This result is also found by trying to implement the torquestiffness curve with the biggest stiffness variation capability from [12]. There, an exponential characteristic was used

$$
\tau(\phi)=-\frac{\ln (-e d \phi)}{e}, \phi<0^{\circ} .
$$

with the real positive constants $e[1 / \mathrm{Nm}]$ and $d[\mathrm{Nm} / \mathrm{rad}]$. If symmetrical cam disc design is assumed $\left(f_{c 1}=f_{c 2}=f_{c}\right.$ and $f_{c 1}^{\prime}=f_{c 2}^{\prime}=f_{c}^{\prime}$, (3) can be reformulated

$$
\tau=4 \operatorname{cr} f_{c} \frac{\partial f_{c}}{\partial \phi} .
$$

Solving this differential equation will result into a cam disc shape with $f_{c}^{\prime}(\phi=0) \neq 0$. So the combination of one spring and two symmetric cam discs to implement the desired characteristic discards.

2) Unsymmetric cam discs: Another possible single spring solution is the combination of one spring with two unsymmetrical cam discs. Unsymmetrical disc shape means here that the minimum of $f_{c}$ is not located at $\phi=0^{\circ}$, see Fig. 5 a). The offset of the minimum from the center is described in the following by $\beta$. In order to achieve a continuous unsymmetrical shape design a piecewise function is used. An example for a cam disc with unsymmetrical disc shape is

$$
f_{c}(\phi)= \begin{cases}R_{1}-\sqrt{\left(R_{1}^{2}-(\phi-\beta)^{2}\right)} & : \phi<\beta \\ R_{2}-\sqrt{\left(R_{2}^{2}-(\phi-\beta)^{2}\right)} & : \phi>\beta,\end{cases}
$$

where $R_{1}$ and $R_{2}$ are different radii. Figure 6 bottom depicts the torque stiffness result for such a piecewise unsymmetrical shape design. The maximum torque for $50 \%$ pretension (dashed line) is approximately $25 \%$ bigger than the maximum torque for the symmetrical shape.

The presented cam disc profile was found by numerically optimizing the maximum torque given by (6) for a pretension 
of $\sigma=50 \%$, bidirectional equal torque and utilizing the operating range of the springs.
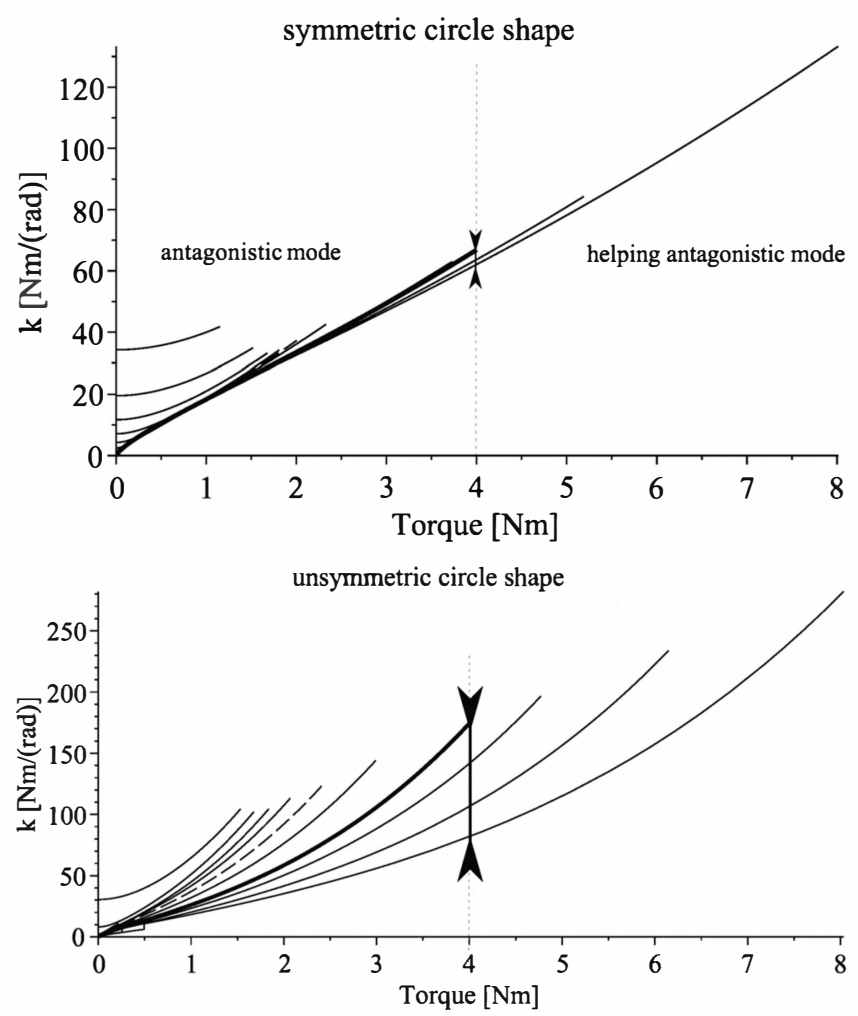

Fig. 6. Single Spring Solution - Top: The symmetric curve design results in a low stiffness for high torques. Bottom: An unsymmetrical curve design extends the stiffness range the helping mode and increases the available joint torque in the normal mode. The border for the helping mode is at $\sigma=26.5 \%$ pretension.

\section{B. Double spring solution}

The limitations of the one spring solution are diminished by using two springs in combination with two unsymmetrical cam discs. However, the setup as it is shown in Fig. 5 b) requires little more space.

The joint torque is calculated by

$$
\begin{array}{r}
\tau=\left(\left(f_{c 1}(\phi+\sigma) f_{c 1}^{\prime}(\phi+\sigma)\right)+\right. \\
\left.\quad\left(f_{c 2}(\phi-\sigma) f_{c 2}^{\prime}(\phi-\sigma)\right)\right) c r
\end{array}
$$

The difference compared to the single spring solution with unsymmetrical shape design and braced spring is that such a mechanism can produce twice the torque (almost half of $\tau_{\max }$ ) at e.g. $50 \%$ pretensioning (see Fig. 7). This is caused by the fact that the maximum spring force of one spring can always be fully used because both cam discs are designed to fully deflect its spring. For the single spring solution at $50 \%$ pretensioning the spring is only deflected half of the maximum deflection. Thus the torque-stiffness bandwidth of this mechanism is increased.

\section{Two springs and four cam discs}

The last setup analyzed contains four cam discs with unsymmetrical shape in combination with two springs (see

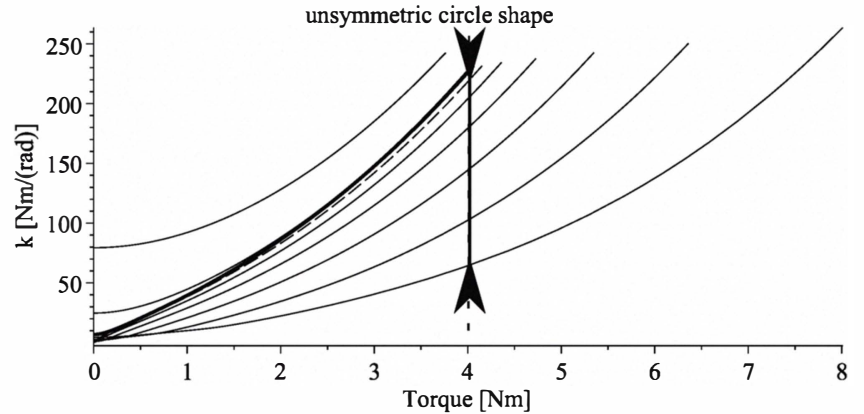

Fig. 7. Torque-stiffness plot for for a BAVS joint with unsymmetrical cam discs and two springs. The border of the helping mode is at $71 \%$ pretension.

Fig. 5 c). The unsymmetrical cam discs allow for a nonzero stiffness even at zero deflection. A separate cam disc is mounted at each of the two circular splines of the harmonic drive gear. The force between each cam disc pair is transmitted over one spring. The requested torque curve must be divided in four identical mirrored curves. The torque is generated through pretensioning of two springs by the cam disc.

$$
\begin{gathered}
\tau=\tau_{1}+\tau_{2} \\
\tau_{1}=\left(f_{c 1}(\phi+\sigma)+f_{c 2}(\phi-\sigma)\right) c \\
\left(f_{c 1}^{\prime}(\phi+\sigma)+f_{c 2}^{\prime}(\phi-\sigma)\right) r \\
\tau_{2}=\left(f_{c 3}(\phi+\sigma)+f_{c 4}(\phi-\sigma)\right) c \\
\left(f_{c 3}^{\prime}(\phi+\sigma)+f_{c 4}^{\prime}(\phi-\sigma) r\right.
\end{gathered}
$$

Figure 8 depicts the resulting exponential torque-stiffness relation obtained by (4) and (5). This approach gives the biggest flexibility for the torque-stiffness shape design.

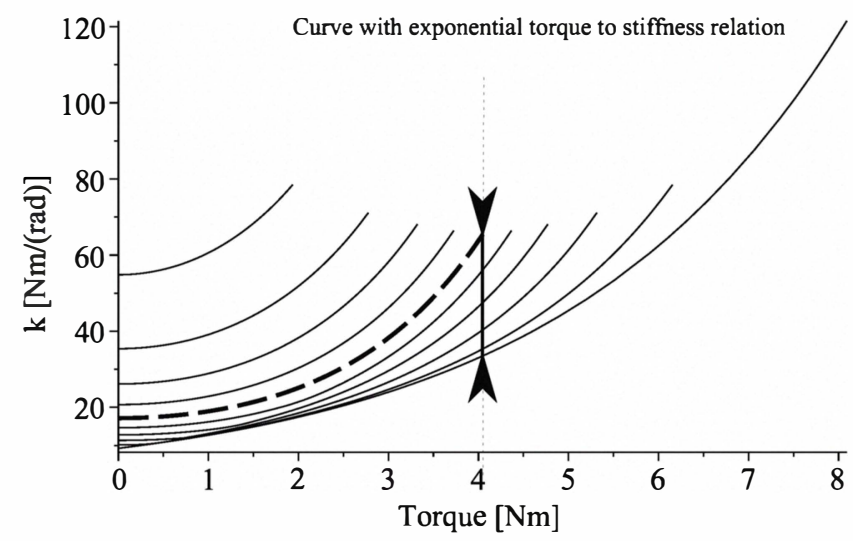

Fig. 8. Exponential torque-stiffness relation for a combination of four cam disc and two springs

Compared to the unsymmetrical circular shape from Fig. (6) the stiffness decreases faster if the cam discs are pretensioned. Which torque-stiffness characteristic is chosen seems to be task depending. So a global answer of the right curve can not be given at this point. 


\section{DESIGN}

\section{A. Design of the Forearm Rotation}

In the forearm rotation a bevel gear system is used instead of a spur gear system in order to achieve an optimal package density (Fig. 9). For the same reason the springs are placed in parallel to the rotational axis of the motors. The cables with cooling water and for controlling the motors of the hand and the complete forearm are guided through a inner hole of the bevel gear wheel in parallel to its rotational axis. Three potentiometers are used to measure the positions of both cam discs and the link side.

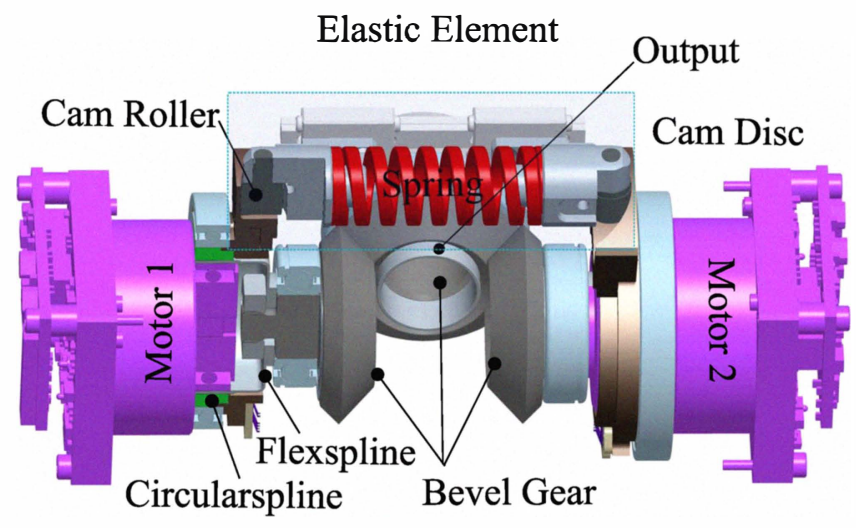

Fig. 9. CAD cross section of the forearm rotation

\section{B. Design of the Wrist}

For the wrist the output bevel wheel is replaced by a guided spur rack in order to place the corresponding motors as near as possible on the proximal site of the forearm. Furthermore the linear springs are placed perpendicular to the rotational axis of the motors. This allows a compact design inside the forearm. Figure 10 shows a cross section of the wrist BAVS drive.

TABLE I

PARAMETERS OF THE TWO BAVS UNITS WITH MOTORS AND ELECTRONICS

\begin{tabular}{ccc} 
Unit & BAVSJ Rotation & BAVSJ Wrist \\
\hline max. Torque [Nm] & 8 & 8 \\
max. Velocity [deg/s] & 960 & 560 \\
Stiffness [Nm/rad] & $0.5-115$ & $0.5-125$ \\
max. stored energy [mJ] & 450 & 700 \\
min. stiffness adjusting time [ms] & 14 & 32 \\
Weight [g] & 960 & 250
\end{tabular}

\section{MEASUREMENT AND EXPERIMENTS}

\section{A. Torque-stiffness shape calibration}

High Quality torque based control of a VS joint requires precise calibration of the nonlinear torque-deflection curve. Here we show measurements of the wrist and forearm BAVS torque-deflection curves. The measurements at the forearm roation were taken using a force gauge mounted on a extra

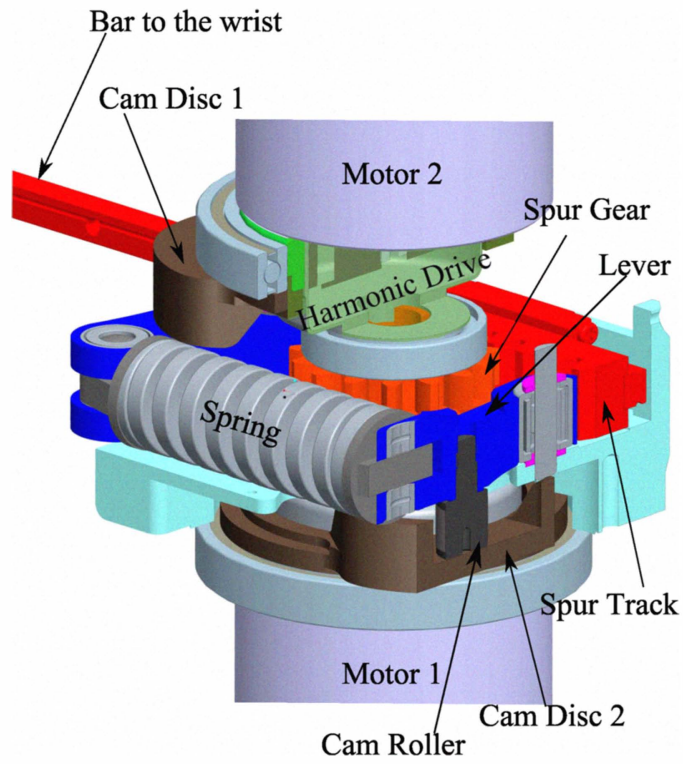

Fig. 10. The picture shows the principle of the wrist actuation. The bended circular spline are connected to the Cam Discs. A movement of the spur results in a rotation of the cam disc, if the motors are blocked. These transfer the torque to the levers over the cam rollers, which compresses the spring.

the lever arm. For the wrist torque to deflection curve a force gauge was direct connected to the bar which actuated the wrist.

In the wrist actuator, a symmetric circle cam disc shape is implemented. The ideal curve and the obtained measurements are depicted in Fig. 11. Only slight deviations exist which arise due to the imperfect stiffness of the connection bar and the force gauge mounting.

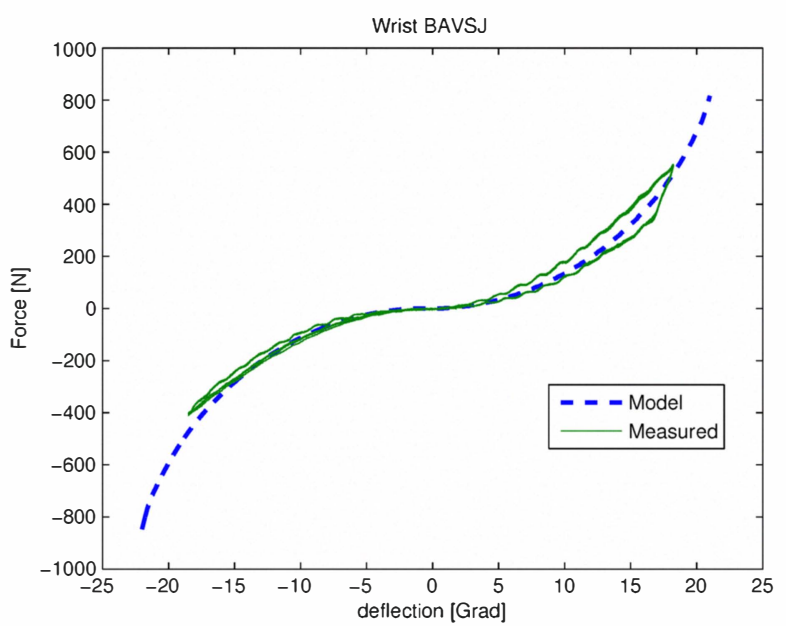

Fig. 11. Computed (blue) and measured (green) torque-deflection relation of the wrist joint.

The forearm rotation torque-displacement curve is shown in Fig. 12 for multiple joint pretensions. The used shape is again a symmetric circle. The decrease of achievable maximum torque is clearly visible. The bigger hysteresis compared to the wrist measurement in Fig. 11 was traced back due to manufacturing tolerance and will be reduced in a future joint version. 


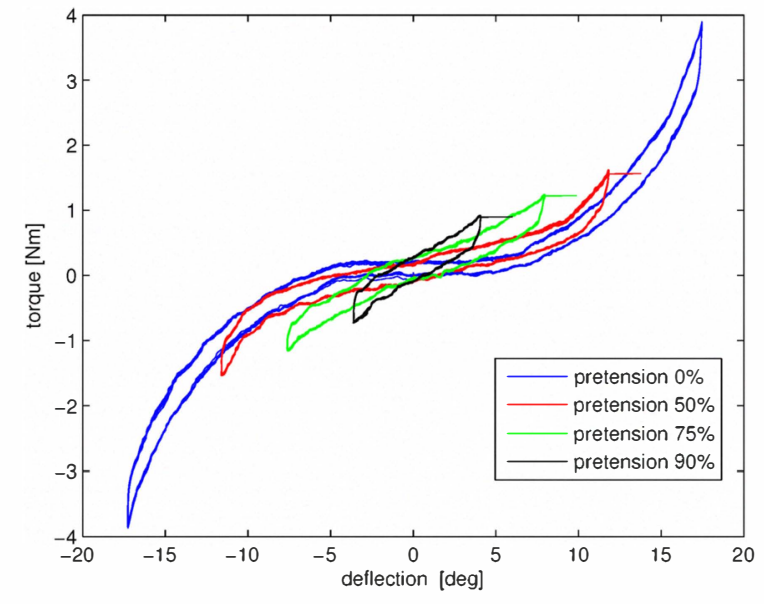

Fig. 12. Torque to deflection curve of the FR with four different pretensions.

\section{B. Automatic stiffness adaption}

If the joint is in the antagonistic mode, the maximum joint torque is half the motor stall torque $\tau_{\text {stall }}$. For some applications it is necessary to reach higher torques by using the helping mode. An algorithm how to transition between normal and helping has been presented in [12]. The approach is to reduce the pretension of the cam disc, when the torque of a motor is too high.

The approach was enhanced for a real time mode change. Within a joint damping is necessary. For every pretension the maximum torque is calculated. If this is crossed, the pretension is reduced linear to the difference of measured torque to maximum torque. The experiment ran on the forearm rotation with a mounted lever arm. The external torque was generated manually. In Fig.13 the torques and stiffnesses are plotted without automatic stiffness adaption. If the stall torque of the first motor is reached, the motor will be driven back. In Fig. 14 the automatic stiffness adaption is activated. If the torque of cam disc one reach $2.4 \mathrm{Nm}$ the second cam disc pretension is reduced

\section{Using redundancy to drive with one motor failure}

A not yet further analyzed property of BAVS joints is the motor redundancy which increases system robustness.

The symmetric setup of the motors realizes the independent generation of motion and stiffness of the joint in a superimposed way. Considering the case only one motor is functional due a failure of the second motor, the stiffness of the joint can not be changed any more but the joint position still can be set. The bidirectional approach allows to drive the malfunctioning motor back. This is a big advantage in autonomous working robots as the failure of one motor does not stop the basic functionality of the robot.

The remaining maximum reachable link torque depends on the stall torque of the running motor minus the torque necessary to drive the gear back of the malfunctioning motor. The knowledge of this torque allows to compute a compensated motor position. Figure 15 shows a plot of BAVS joint in position control with one motor switched off. The
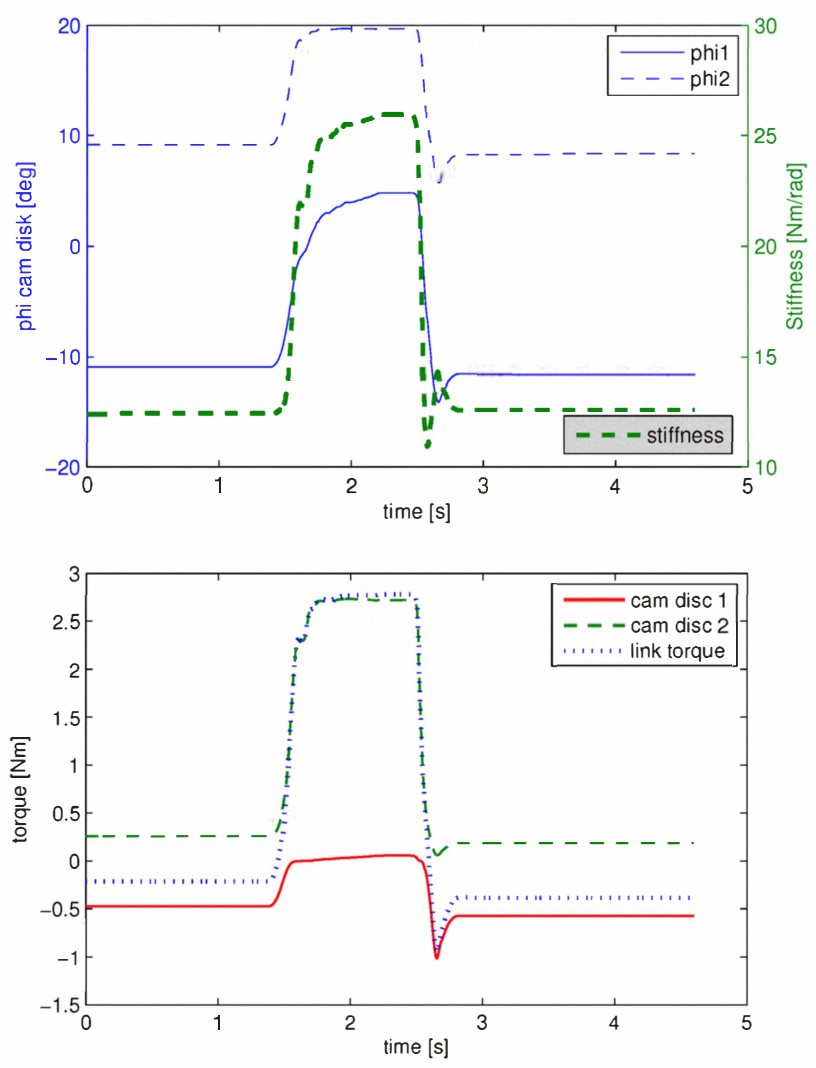

Fig. 13. This plot shows joint measurements for an external disturbance without automatic stiffness adaption. On the top the cam disc angles and the joint stiffness is shown. The bottom figure shows the motor torques.

used computed position feed forward term is the necessary deflection of the cam disc to achieve the backdrive torque.

$$
\begin{gathered}
q_{\text {drive }}=q_{\text {des }}+q_{\text {backdrive }} \\
q_{\text {backdrive }}=\tau_{\text {camdisc }}^{-1}\left(\tau_{\text {back }}(\omega)\right)
\end{gathered}
$$

The backdrive torque is increasing with higher link velocities. The back drive moment was documented on an extra test bed while the efficiency of the gear was measured.

\section{CONCLUSIONS AND FUTURE WORKS}

The hardware realization of bidirectional antagonistic joints using an harmonic drives mechanism for the DLR Hand Arm System is presented in this paper. Three main design concepts with different spring and cam disc setups have been evaluated and optimized for torque capability and range of stiffness, especially during the helping mode. The mechanical design of the wrist and forearm rotation joint of the DLR Hand Arm System is presented. Measurements of the joint calibration and two experiments on the realized joints are shown. First, an automatic stiffness adaption scheme was implemented to provide always the maximum torque. Second, the advantage of motor redundancy in the case of motor failure is presented and evaluated. In future work, the unsymmetric shape design with the calculated increase in bandwidth of the stiffness-torque curve will be implemented and evaluated. Furthermore, the energy consumption of the system has to be evaluated for different 

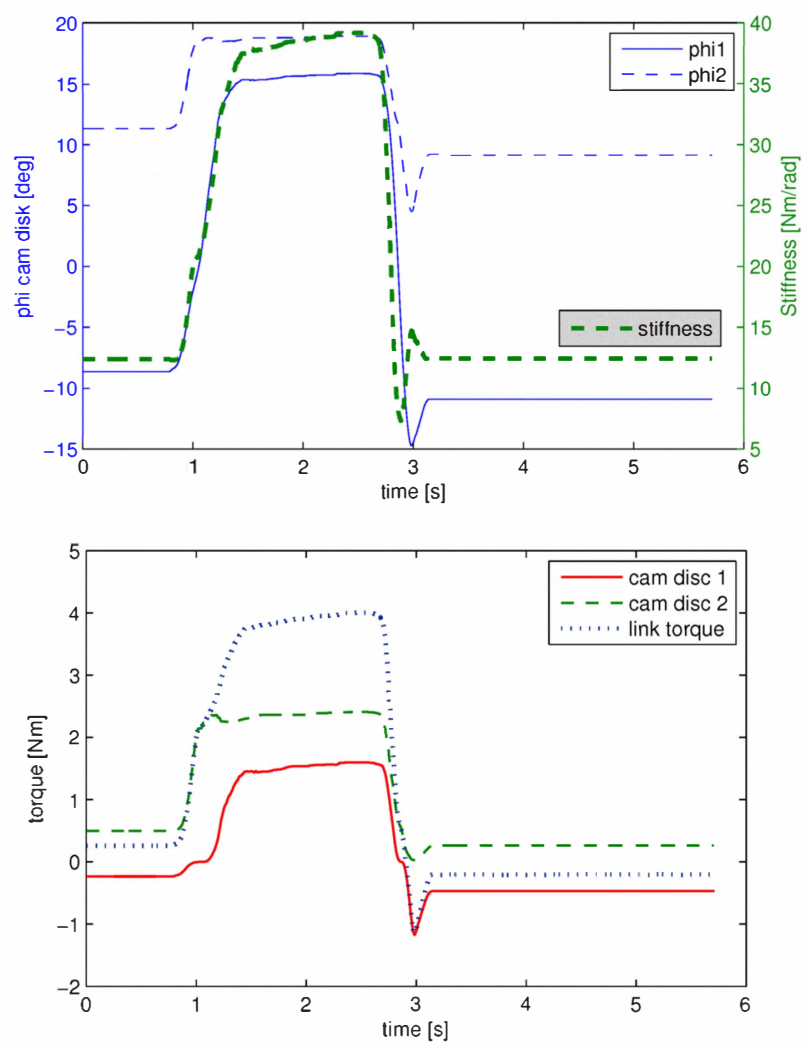

Fig. 14. Measurements of the joint for an external disturbance with activated stiffness adaptation. The adaptation of the pretension of the joint allows to reach higher torques and stiffness.

tasks if the link torque is shared variable on both motors. The results obtained from task execution in the DLR Hand Arm system are expected to offer valuable results for the torque-stiffness shape design.

\section{ACKNOWLEDGMENTS}

This work was partially funded by the European Commissions Sixth Framework Program as part of the project STIFF under grant no. 231576 and the project VIACTORS under grant no. 231554.

\section{REFERENCES}

[1] M. Grebenstein, A. Albu-Schaffer, T. Bahls, M. Chalon, O. Eiberger, W. Friedl, R. Gruber, U. Hagn, R. Haslinger, H. Hoppner, S. Jorg, M. Nickl, A. Nothhelfer, F. Petit, J. Reill, N. Seitz, T. Wimbock, S. Wolf, T. Wusthoff, and G. Hirzinger, "The dlr hand arm system," in in Proceedings of the 2011 IEEE International Conference on Robotics and Automation, 2011.

[2] J. W. Hurst, J. E. Chestnutt, and A. A. Rizzi, "An actuator with physically variable stiffness for highly dynamic legged locomotion," in Proc. IEEE Int. Conf. Robotics and Automation ICRA '04, vol. 5, 2004, pp. 4662-4667.

[3] B.-S. Kim and J.-B. Song, "Hybrid dual actuator unit: A design of a variable stiffness actuator based on an adjustable moment arm mechanism," in Proc. IEEE Int Robotics and Automation (ICRA) Conf, 2010, pp. 1655-1660.

[4] R. Schiavi, G. Grioli, S. Sen, and A. Bicchi, "Vsa-ii: a novel prototype of variable stiffness actuator for safe and performing robots interacting with humans," in Proc. IEEE Int. Conf. Robotics and Automation ICRA 2008, 2008, pp. 2171-2176.

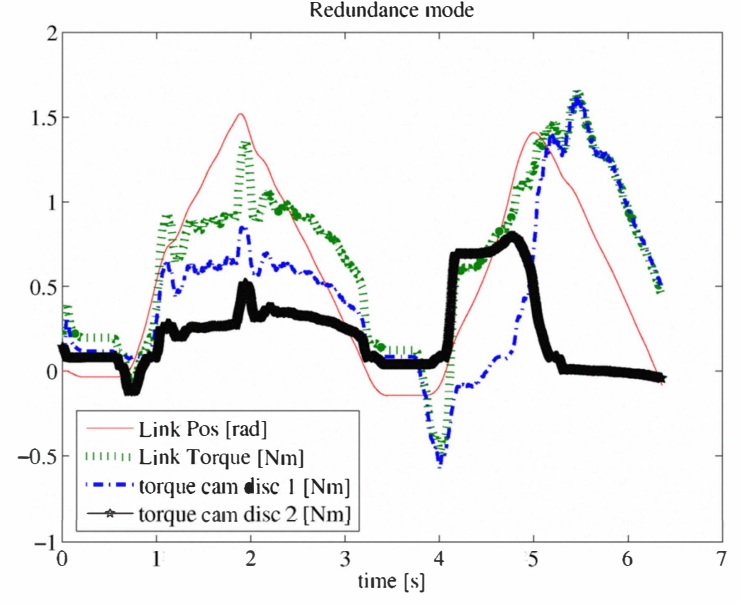

Fig. 15. Both motor are running until at $\mathrm{t}=4 \mathrm{~s}$ one motor is switched off (failure motor). When the torque necessary to drive the failure motor is reached, the motor motion of the running motor is transmitted to the output.

[5] R.-J. Wang and H.-P. Huang, "An active-passive variable stiffness elastic actuator for safety robot systems," in Proc. IEEE/RSJ Int Intelligent Robots and Systems (IROS) Conf, 2010, pp. 3664-3669.

[6] J. Kobayashi, K. Okumura, Y. Watanabe, and N. Suzuki, "Development of variable stiffness joint drive module and experimental results of joint angle control," in Proc. of The Fifteenth International Symposium on Artificial Life and Robotics, 2010, pp. 946-949.

[7] V. Q. Hung, L. Aryananda, F. I. Sheikh, F. Casanova, and R. Pfeifer, "Novel mechanism for varying stiffness via changing transmission angle," in Proc. IEEE Int Robotics and Automation (ICRA) Conf, 2011.

[8] S. Haddadin, A. Albu-Schaffer, O. Eiberger, and G. Hirzinger, "New insights concerning intrinsic joint elasticity for safety," in Proc. IEEE/RSJ Int Intelligent Robots and Systems (IROS) Conf, 2010, pp. 2181-2187.

[9] [Online]. Available: www.viactors.org

[10] M. Grebenstein and P. van der Smagt, "Antagonism for a highly anthropomorphic handarmsystem," Advanced Robotics, vol. 22, pp. $39-55,2008$.

[11] S. Wolf, O. Eiberger, and G. Hirzinger, "The DLR FSJ: energy based design of a variable stiffness joint," in Proc. IEEE Int. Conf. Robotics and Automation ICRA 2011, 2011.

[12] F. Petit, M. Chalon, W. Friedl, M. Grebenstein, A. A. Schaeffer, and G. Hirzinger, "Bidirectional antagonistic variable stiffness actuation: Analysis, design \& implementation," in Proc. IEEE Int Robotics and Automation (ICRA) Conf, 2010, pp. 4189-4196.

[13] N. Gialias and Y. Matsuoka, "Muscle actuator design for the act hand," in Proc. IEEE Int. Conf. Robotics and Automation ICRA '04, vol. 4, 2004, pp. 3380-3385. 